\title{
Thermal and magnetic evolution of a crystallizing basal magma ocean in Earth's mantle
}

\author{
Nicolas A. Blanc ${ }^{\mathrm{a}}$, Dave R. Stegman ${ }^{\mathrm{a}}$, Leah B. Ziegler ${ }^{\mathrm{a}}$ \\ ${ }^{a}$ Institute for Geophysics and Planetary Physics, Scripps Institution of Oceanography, \\ University of California San Diego, La Jolla, California, USA
}

\begin{abstract}
We present the thermochemical evolution of a downward crystallizing BMO overlying the liquid outer core and probe its capability to dissipate enough power to generate and sustain an early dynamo. A total of 61 out of 112 scenarios for a BMO with imposed, present-day $Q_{B M O}$ values of 15,18 , and 21 TW and $Q_{r}$ values of 4, 8, and 12 TW fully crystallized during the age of the Earth. Most of these models are energetically capable of inducing magnetic activity for the first 1.5 Gyrs, at least, with durations extending to 2.5 Gyrs; with final CMB temperatures of $4400 \pm 500 \mathrm{~K}$-well within current best estimates for inferred temperatures. None of the models with $Q_{B M O}=$ 12 TW achieved a fully crystallized state, which may reflect a lower bound on the present-day heat flux across the CMB. BMO-powered dynamos exhibit strong dependence on the partition coefficient of iron into the liquid layer and its associated melting-point depression for a lower mantle composition at near-CMB conditions -parameters which are poorly constrained to date. Nonetheless, we show that a crystallizing BMO is a plausible mechanism to sustain an early magnetic field.
\end{abstract}

Email address: nblanc@ucsd.edu () 
Keywords: Basal magma ocean dynamo, Early Earth dynamo, Molten silicates

\section{1. Introduction}

2 A fundamental constraint on the thermal evolution of the Earth is that 3 of the presence of a magnetic field since at least 3.45 Ga (Tarduno et al., 4 2010), and possibly even since $4.2 \mathrm{Ga}$ (Tarduno et al., 2015). Some recent 5 estimates on the thermal conductivity of the Earth's core imply estimates of 6 core heat flow on the order of 15 TW (de Koker et al., 2012; Pozzo et al., 7 2012; Gomi et al., 2013) which favors a young (<500 Myr) inner core (Ol8 son, 2013; Davies, 2015; Labrosse, 2015; Nimmo, 2015). Since the buoyancy 9 sources associated with inner core nucleation (light element concentration and latent heat) are the main sources of power dissipation for magnetic activity generation for the current field (Buffett et al., 1996; Stevenson, 2003), generating a geodynamo in the absence of an inner core (through secular cooling of the core) poses significant challenges (Olson, 2013). Moreover, thermal evolution models that incorporate high core heat flow, such as those implied by higher thermal conductivity values, also imply extensive melting of the mantle (i.e. a "thermal catastrophe") (Korenaga, 2013, 2008; Driscoll and Bercovici, 2014).

Sustaining a dynamo for $>3$ Gyrs in the absence of an inner core led to the proposal of alternative mechanisms for powering a geodynamo, such as the exsolution of light material across the core mantle boundary (CMB) (ORourke and Stevenson, 2016; O’Rourke et al., 2017; Badro et al., 2016; Hi-

rose et al., 2017). Experimental determination of exsolution reactions (Badro 
et al., 2016; Hirose et al., 2017; Badro et al., 2018) indicate this may be a viable mechanism, however, there are still questions as to whether either would provide sufficient power, duration, or be active during the period of interest (Du et al., 2017, 2019; Badro et al., 2018).

Exsolution mechanisms explicitly occur across a metal-silicate interface that is liquid on both sides (Badro et al., 2016, 2018), thus invoking a longlived basal magma ocean (BMO) atop the core (Labrosse et al., 2007; Laneuville et al., 2018) which would initiate at mid-mantle depths and crystallize downwards to the core. A giant impact as large as one suggested to lead to the formation of the Moon may have been energetic enough that Earth's initial condition was completely molten (Canup and Asphaug, 2001; Ćuk and Stewart, 2012; Lock et al., 2018), however the initial depth of an emergent $\mathrm{BMO}$ is subject to uncertainty in the equation of state of lower mantle composition, its melting curve, the adiabatic gradient as determined by its material properties, and the dynamics of phase separation (Stixrude et al., 2009; De Koker and Stixrude, 2009; Boukaré et al., 2015; Boukaré and Ricard, 2017; Wolf and Bower, 2018; Caracas et al., 2019). The scenario of whether the BMO, if electrically conductive enough, could be capable of generating a dynamo was explored as a potential mechanism for providing a magnetic field during the early Earth (Ziegler and Stegman, 2013). Recent theoretical calculations of the electrical conductivity of molten silicates at P-T conditions appropriate for the $\mathrm{CMB}$ report values that support it as a material that is sufficiently electrically conductive (Spaulding et al., 2012; McWilliams et al., 2012; Soubiran and Militzer, 2018; Holmström et al., 2018; Stackhouse et al., 2010; Scipioni et al., 2017). 
The previous conceptual model for a BMO-powered dynamo (Ziegler and Stegman, 2013) used an idealized phase diagram for the evolution of a crystallizing basal magma ocean (Labrosse et al., 2007). In this study, we present the thermochemical evolution of a downward crystallizing BMO (see Figure 1) using recent thermodynamic and mineral physics for molten silicates at lower mantle P-T conditions (Stixrude, 2014; Boukaré et al., 2015; Caracas et al., 2019), and their associated entropy budgets provide a more robust measure of evaluating the circumstances under which the BMO played a role in the magnetic evolution of early Earth. We constrain our models with the early magnetic history of Earth and estimates of the current CMB temperature.

\section{Model and Methods}

We build upon an established theory for gross thermodynamics of the Earth's core with a solidifying inner core (Gubbins et al., 2003, 2004; Nimmo, 2015) and apply it to the scenario of a downward crystalizing BMO layer overlying Earth's core. We adapt an existing general 1D model for thermochemical evolution of the Earth's core (Davies, 2015) to study the evolution and fractional crystallization of an FeO-enriched basal magma ocean (see Fig. 5C, D in (Caracas et al., 2019) for reference). We approximate the molten layer to be a fluid in hydrostatic equilibrium with an adiabatic temperature where a homogenously-mixed composition is maintained by vigorous convection everywhere outside thin boundary layers.

The global energy budget for the BMO-layer determines its evolution by balancing the heat flux across the top of the layer $\left(Q_{B M O}\right)$ against the sum 
of all heat sources within the layer. While the energy budget contains information about the cooling rate of the layer and, inevitably, the rate at which it crystallizes, it lacks information about the dynamo as all magnetic energy is converted into heat within the layer. Dynamo information is inherently embedded in the entropy budget equations which relate all entropy sources to the two most significant entropy sinks, thermal diffusion and Ohmic dissipation; the power available to drive a dynamo is related to the latter. Combining the energy budget with the associated entropy budget provides sufficient information to describe and characterize the thermal and magnetic evolution of the BMO over time.

\subsection{Energy Budget}

The total energy, $Q_{B M O}$, extracted through the top of the BMO layer by the overlying solid mantle is the sum of all energy sources within the layer. The complete energy budget can be written as

$$
Q_{B M O}=Q_{s}+Q_{g}+Q_{L}+Q_{r}+Q_{P}+Q_{H}+Q_{C M B}
$$

which includes the secular cooling of the layer, $Q_{s}$, the gravitational potential energy released during solidification, $Q_{g}$, the latent heat generated as the layer solidifies, $Q_{L}$, the heat due to radioactive decay, $Q_{r}$, the heat due to a change in pressure due to thermal contraction, $Q_{P}$, and the heat of reaction, $Q_{H}$. The contribution from $Q_{P}, Q_{H}$ is negligible, they are only included for completeness; and $Q_{C M B}$ is heat attributed to the cooling of the core. Following (Gubbins et al., 2003, 2004; Davies, 2015), the first four terms except for $Q_{r}$ can be related to the cooling rate, $d T_{r}^{B M O} / d t$, where $T_{r}^{B M O}$ is the temperature of the layer at the solidification front radius, $r$. 
Indeed, these terms have been previously derived for the case of the solidifying inner core at length elsewhere (Gubbins et al., 2003, 2004; Nimmo, 2015; Davies, 2015), thus we briefly summarize their analytical expressions below and, where necessary, explain the modification made in adapting these formulations to better represent the BMO scenario.

The first term in Eq. 1 describes the energy associated with the secular cooling of the layer and it can be expressed as

$$
Q_{s}=-\int \rho C_{p} \frac{d T_{r}^{B M O}}{d t} d V
$$

where $\rho$ and $C_{p}$ are the density and specific heat capacity of the layer, respectively. This term is simply the amount of heat released as the layer cools volumetrically. The second term in 1 is related to the amount of gravitational energy released due to the re-distribution of lighter elements to the top of the layer, or equivalently the displacement of denser elements to the bottom of the layer, upon crystallization. It is given by

$$
Q_{g}=\int \rho \psi \alpha_{B M O} \frac{D c}{D t} d V
$$

where $\psi$ is the gravitational potential. The parameter $\alpha_{B M O}$ is a dimensional coefficient which specifies the sensitivity of the layer density to the enrichment of $\mathrm{FeO}$, analogous to $\alpha_{c}$ described in (Gubbins et al., 2004) due to the presence of light elements in the core. It is given by

$$
\alpha_{B M O}=-\frac{1}{\rho}\left(\frac{\partial \rho}{\partial c}\right)_{P, T} \approx \frac{\Delta \rho_{B M O}}{\rho_{r}^{B M O} \Delta c_{F e O}^{l}}
$$

where $\rho_{r}^{B M O}$ is the density of the BMO layer at the solid-liquid interface radius $r$, and $\Delta \rho_{B M O}$ is the density jump across the interface due to the 
change in concentration of the liquid, $\Delta c_{F e O}^{l}$, as it becomes progressively enriched in $\mathrm{FeO}$ upon solidification.

The change in concentration dependents on the rate at which $\mathrm{FeO}$ is incorporated into the solidifying bridgmanite phase which is controlled entirely by the partitioning coefficient, $D_{\mathrm{FeO}}$. This allows for the following expression, $\Delta c_{F e O}^{l}=c_{F e O}^{l}\left(1-D_{F e O}\right)$ which relates the amount of $\mathrm{FeO}$ in the liquid, $c_{F e O}^{l}$, to its partitioning coefficient. We estimate values for $\alpha_{B M O}$ (see Table 2) using $\Delta \rho_{B M O}=200-300 \mathrm{~kg} / \mathrm{m}^{3}$ from (Caracas et al., 2019) and two different partition coefficients for $\mathrm{FeO}: D_{F e O}=0.1$ (Nomura et al., 2011) and $D_{\mathrm{FeO}}=0.5$ (Andrault et al., 2012).

Lastly, the amount of gravitational energy released at the interface depends on the rate at which lighter elements are re-distributed to the top of the layer, Dc/Dt. Following (Gubbins et al., 2004), we relate this term to the rate at which the interface crystallizes,

$$
\frac{D c}{D t}=C c \frac{d r_{t o p}}{d t}=\frac{4 \pi r_{t o p}^{2} \rho_{t o p} \Delta c}{M_{r}^{B M O}},
$$

where $r_{\text {top }}$ and $\rho_{\text {top }}$ are the radius and the density at the top of the BMO layer, $\Delta c$ is the change in concentration of the liquid layer, and $M_{r}^{B M O}$ is the mass of the liquid layer.

The third term in 1 accounts for the latent heat generated and released at the interface as the layer solidifies and it depends on the rate at which this process occurs:

$$
Q_{L}=4 \pi r_{\text {top }}^{2} L_{H} \rho_{\text {top }} \frac{d r_{\text {top }}}{d t},
$$

where $L_{H}$ is the latent heat of reaction which is assumed to be constant. The last term in 1 is simply the heat generated within the volume of the BMO 
layer by the decay of radioactive elements. Considering a density $\rho$ and a volumetric heating rate, $h$, over a volume, $V$, this term can be written as

$$
Q_{r}=\int \rho h d V
$$

The heat production is time-dependent according to the assumed BSE concentrations, long-lived radioactive decay energies and halflives, which are given in Table 1. Making use of the formulations presented above, the total energy budget 1 for a crystallizing BMO layer can be expressed as follows

$$
\begin{aligned}
Q_{B M O} & =-\int \rho C_{p} \frac{d T_{r}^{B M O}}{d t} d V+\int \rho \psi \alpha_{B M O} \frac{D c}{D t} d V \\
& +4 \pi r_{\text {top }}^{2} L_{H} \rho_{t o p} \frac{d r_{t o p}}{d t}+\int \rho h d V+M_{\text {core }} C_{p(\text { core })} \frac{d T_{r}^{B M O}}{d t} \\
& =Q_{s}+Q_{g}+Q_{L}+Q_{r}+Q_{C M B}
\end{aligned}
$$

\subsection{BMO solidification model}

In this work, we consider a BMO layer overlying the liquid core crystallizing from the top down towards the CMB, whose thickness is determined by the intersection of the adiabat and the melting curve as shown schematically in Figure 1. As the layer cools, the adiabat intersects the melting curve at greater depths causing the layer to shrink. The initial thickness of the layer is determined by the initial temperature; here, we define two different values for the initial temperature of the layer resulting in two initial thicknesses: $r_{0}^{t o p}=4242 \mathrm{~km}$ and $r_{0}^{t o p}=4458 \mathrm{~km}$.

We implement a lower mantle adiabat with two different gradients, and utilize the melting curve for a peridotite mantle composition (Fiquet et al., 2010; Stixrude, 2014). Two different melting point depressions at the CMB, $T_{m}^{0}=700 \mathrm{~K}$ and $T_{m}^{0}=1100 \mathrm{~K}$ are imposed onto the undepressed melting 


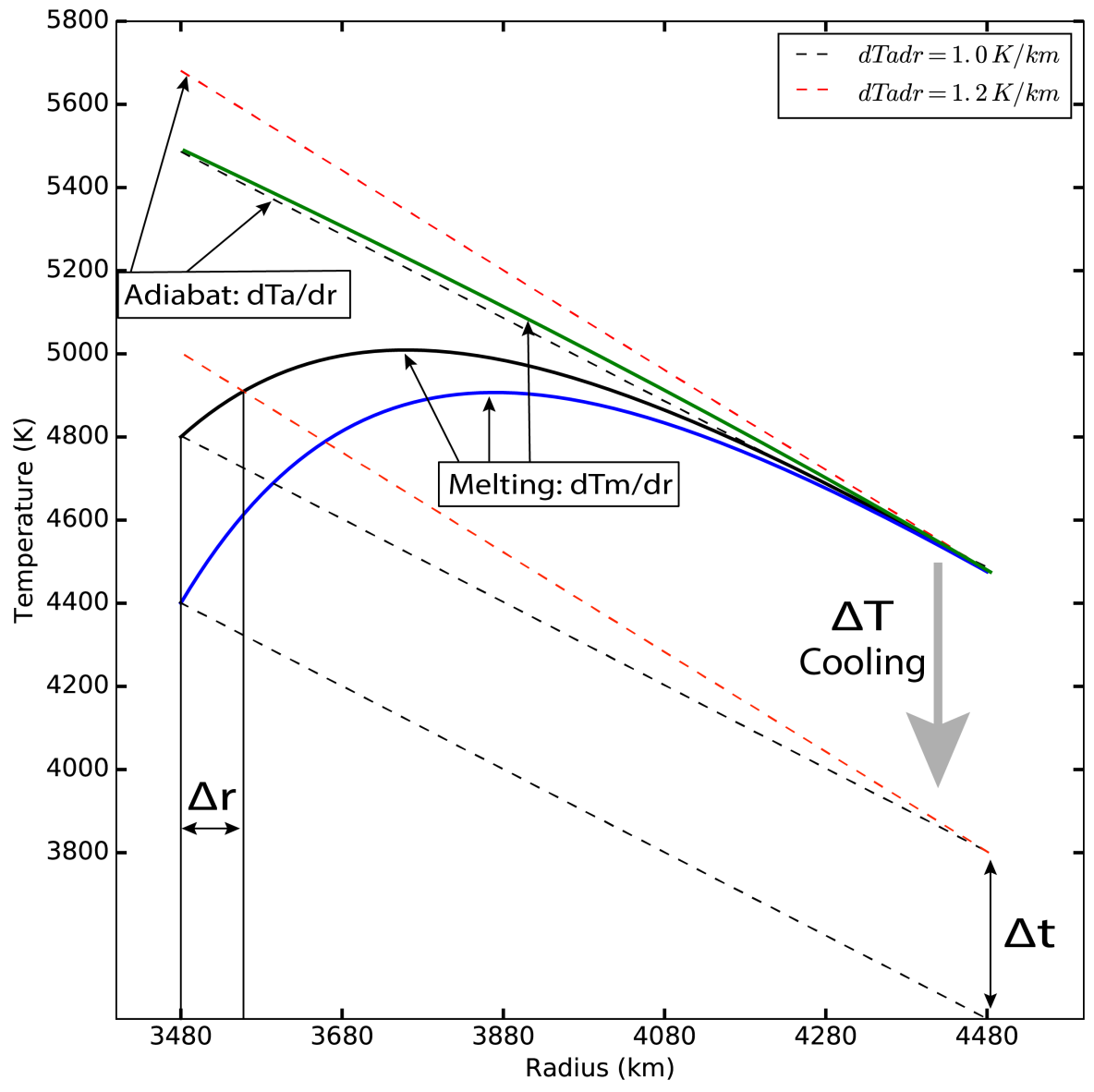

Figure 1: BMO thermal evolution diagram. The thickness of the BMO layer is defined by the intersection of the adiabat, $T_{a}$, and the melting curve, $T_{m}$. The corresponding temperature at the CMB is determined by tracking the adiabat to the appropriate radius. Secular cooling of the layer over time moves the adiabat to lower temperatures, intersecting the melting curve at greater depths thus crystallizing the layer downward toward the CMB. Imposing a larger melting point depression at the CMB to the melting curve (black versus blue solid curves) prolongs the life of the BMO layer as it is required to cool an additional amount of time, $\Delta t$, to fully crystallize. Moreover, at a time, $t$, two different adiabats (red and black dashed lines) will intersect a given melting curve at different points changing the thickness of the BMO layer at that point in time by an amount $\Delta r$. 
Table 1: Input parameters.

\begin{tabular}{|c|c|c|c|c|}
\hline Definition & Symbol & Units & Value & Reference \\
\hline Density & $\rho$ & $\mathrm{Kg} \mathrm{m}^{-3}$ & & (Dziewonski and Anderson, 1981) \\
\hline Density jump & $\Delta \rho_{B M O}$ & $\mathrm{Kg} \mathrm{m}^{-3}$ & $200-300$ & (Caracas et al., 2019) \\
\hline CMB pressure & & $\mathrm{GPa}$ & 135 & \\
\hline Mantle specific heat & $C_{p_{\text {mantle }}}$ & $\mathrm{J} \mathrm{Kg}^{-1} \mathrm{~K}^{-1}$ & 1000 & (Labrosse et al., 2007) \\
\hline Core specific heat & $C_{p_{\text {core }}}$ & $\mathrm{J} \mathrm{Kg}^{-1} \mathrm{~K}^{-1}$ & 860 & (Labrosse et al., 2007) \\
\hline Mass of Core & $M_{\text {core }}$ & $\mathrm{Kg}$ & $2 \times 10^{24}$ & (Labrosse et al., 2007) \\
\hline Adiabatic gradient & $d T_{a} / d r$ & $\mathrm{~K} / \mathrm{km}$ & $1.0 / 1.2$ & \\
\hline Entropy of melting per unit mass & $\Delta s$ & $\mathrm{~J} \mathrm{Kg}^{-1} \mathrm{~K}^{-1}$ & 300 & (Labrosse et al., 2007) \\
\hline Melting temperature at the $\mathrm{CMB}$ & & $\mathrm{K}$ & 5400 & (Fiquet et al., 2010) \\
\hline Melting-point depression at the CMB & $T_{m}^{0}$ & $\mathrm{~K}$ & $700 / 1000$ & \\
\hline Thermal conductivity of the mantle & $k$ & $\mathrm{~W} \mathrm{~m}^{-1} \mathrm{~K}^{-1}$ & 8 & (Labrosse et al., 2007) \\
\hline Initial $\mathrm{FeO}$ concentration & & wt $\%$ & 15.99 & (Caracas et al., 2019) \\
\hline $\mathrm{FeO}$ partition coefficient & $D_{F e O}$ & & $0.1 / 0.5$ & (Nomura et al., 2011; Andrault et al., 2012) \\
\hline
\end{tabular}

curve (green curve in Figure 1) to represent the depression induced by the progressive enrichment of the liquid layer in $\mathrm{FeO}$ as it crystallizes. The density of the layer is estimated to be similar to that of the current lower mantle for which we utilize a polynomial fit to the Preliminary reference Earth model (PREM) (Dziewonski and Anderson, 1981).

We take the initial concentration of $\mathrm{FeO}$ in the liquid to be that of (Caracas et al., 2019) for a pyrolitic melt after $50 \%$ crystallization of bridgmanite and allow it to evolve using two different (end-member) values for the partition coefficient: $D_{\mathrm{FeO}}=0.5$ (Andrault et al., 2012), and $D_{\mathrm{FeO}}=0.1$ (Nomura et al., 2011). However, since the behavior of $D_{F e O}$ has only been characterized for Fe partitioning between bridgmanite and liquid compositions which have relatively modest Fe concentrations, we would expect $D_{F e O}$ to deviate from these measured values once the system has evolved to be heavily $\mathrm{Fe}$ enriched. Accordingly, we adopt a conservative threshold at $c_{\mathrm{FeO}}^{l}=50 \mathrm{wt} \%$ for the interpretation of our model results once the concentration of $\mathrm{FeO}$ in 
the liquid exceeds $50 w t \%$, as we anticipate the partition coefficient for such a state to deviate from the constant value being applied. All input parameters are presented in Table 1.

The evolution of all models is largely controlled by the amount of heat being extracted by the solid mantle from the top of the BMO layer and the amount of radiogenic heat produced within the layer. Henceforth we refer to the combination of these two parameters as "cooling history". The internal heating of the mantle corresponds to a Bulk Silicate Earth (BSE) model (McDonough and Sun, 1995) comprises of the decay energies for the 4 long lived radioactive isotopes (U235, U238, Th232, and K40) in the appropriate ratios. The radiogenic heat production over time is prescribed by the sum of the abundance and decay energies for the 4 isotopes and their corresponding half-lives, based upon the present day heat production for the BSE of 20 TW. Approximately $8 \mathrm{TW}$ of the total BSE complement is assumed to reside in the continental crust, and the remaining $12 \mathrm{TW}$ (out of the $20 \mathrm{TW}$ total) therefore resides within the mantle, for which we consider 3 scenarios $(4,8$ or $12 \mathrm{TW}$ ) for how much is contained within the BMO, representing $33 \%$, $67 \%$, or $100 \%$ of the available heat production shown as dashed curves in Figure 2. The complement of radiogenic heat producing elements initially in the BMO are assumed to remain in the BMO for its entire evolution, and thus for models that have completely solidified, the entirety of the radiogenic heating would be contained within a very thin layer in the mantle atop the CMB.

The cooling of the BMO, $Q_{B M O}(t)$, is controlled by a cooling history that is prescribed at the interface between solid and liquid mantle with a present- 
Table 2: Model results from all the different $\mathrm{Q}_{B M O} / \mathrm{Q}_{r}$ setups probed in this study. The input parameters for each run (1-16) within a given setup are the adiabatic gradient $(d T a / d r)$ in $\mathrm{K} / \mathrm{km}$, the partition coefficient for $\mathrm{FeO}\left(D_{F e O}\right)$, compositional coefficient $\left(\alpha_{B M O}\right)$, and the imposed depression on the melting curve $\left(T_{m}^{0}\right)$. Given our conservative cutoff, we report the available entropy due to Ohmic dissipation $\left(\mathrm{E}_{\Phi}\right)$ at $c_{F e O}^{l}=50 w t \%$ in $\mathrm{MW} / \mathrm{K}$, and the time when this cutoff is reached $\left(\mathrm{t}_{X}\right)$, along with the time when $\mathrm{E}_{\Phi}$ falls below zero. The time it takes each model to fully crystallize (if successful) is given by $\mathrm{t}_{B M O}$ and the final temperature at the CMB for each case is given by $\mathrm{T}_{C M B}^{f i n a l}$.

\begin{tabular}{|c|c|c|c|c|c|c|c|c|c|c|c|c|c|c|c|c|}
\hline COMBO \# & 1 & 2 & 3 & 4 & 5 & 6 & 7 & 8 & 9 & 10 & 11 & 12 & 13 & 14 & 15 & 16 \\
\hline$d T a / d r$ & 1 & 1 & 1 & 1 & 1 & 1 & 1 & 1 & 1.2 & 1.2 & 1.2 & 1.2 & 1.2 & 1.2 & 1.2 & 1.2 \\
\hline$D_{\mathrm{FeO}}$ & 0.1 & 0.1 & 0.1 & 0.1 & 0.5 & 0.5 & 0.5 & 0.5 & 0.1 & 0.1 & 0.1 & 0.1 & 0.5 & 0.5 & 0.5 & 0.5 \\
\hline$\alpha_{B M O}$ & 0.3 & 0.3 & 0.4 & 0.4 & 0.4 & 0.4 & 0.3 & 0.3 & 0.3 & 0.3 & 0.4 & 0.4 & 0.4 & 0.4 & 0.3 & 0.3 \\
\hline$T_{m}^{0}$ & 700 & 1100 & 700 & 1100 & 700 & 1100 & 700 & 1100 & 700 & 1100 & 700 & 1100 & 700 & 1100 & 700 & 1100 \\
\hline \multicolumn{17}{|c|}{ Cooling history model: $\star \mathrm{Q}_{B M O}^{\text {present }}=15 \mathrm{TW}, \mathrm{Q}_{r}=4 \mathrm{TW}, \mathrm{Q}_{C M B}^{\text {present }}=11 \mathrm{TW}$} \\
\hline $\mathrm{E}_{\Phi}$ & 280 & 215 & 351 & 21 & 1 & -30 & -9 & -35 & 265 & 182 & 324 & 222 & -40 & -80 & -50 & -83 \\
\hline $\mathrm{t}_{X}(\mathrm{Myr})$ & 1050 & 1390 & 1070 & 1430 & 1690 & 2550 & 1670 & 2520 & 1540 & 1860 & 1600 & 1920 & 2600 & 3590 & 2560 & 3540 \\
\hline $\mathrm{t}_{\Phi}(\mathrm{Myr})$ & 2200 & 2810 & 2370 & 3080 & 1690 & $2210^{*}$ & $1620^{*}$ & $2120^{*}$ & 2920 & 3160 & 3240 & 3470 & $2250^{*}$ & $2560^{*}$ & $2140^{*}$ & $2450^{*}$ \\
\hline $\mathrm{t}_{B M O}(\mathrm{Myr})$ & 2420 & 4070 & 2480 & 4180 & 2330 & 3930 & 2310 & 3890 & 4020 & & 4180 & & 3820 & & 3750 & \\
\hline $\mathrm{T}_{C M B}^{f i n a l}(\mathrm{~K})$ & 4258 & 4310 & 4279 & 4334 & 4228 & 4279 & 4220 & 4270 & 4500 & 4433 & 4533 & 4458 & 4453 & 4401 & 4438 & 4390 \\
\hline \multicolumn{17}{|c|}{ Cooling history model: $\mathbf{Q} \mathrm{Q}_{B M O}^{\text {present }}=18 \mathrm{TW}, \mathrm{Q}_{r}=4 \mathrm{TW}, \mathrm{Q}_{C M B}^{\text {present }}=14 \mathrm{TW}$} \\
\hline $\mathrm{E}_{\Phi}$ & 453 & 360 & 566 & 434 & 49 & 15 & 34 & 6 & 484 & 364 & 584 & 435 & 18 & -24 & 7 & -32 \\
\hline $\mathrm{t}_{X}(\mathrm{Myr})$ & 750 & 960 & 760 & 980 & 1160 & 1620 & 1150 & 1610 & 1060 & 1250 & 1100 & 1280 & 1690 & 2170 & 1660 & 2150 \\
\hline $\mathrm{t}_{\Phi}(\mathrm{Myr})$ & 1560 & 2100 & 1610 & 2250 & 1300 & 1700 & 1250 & 1640 & 2200 & 2500 & 2390 & 2700 & 1760 & $2050^{*}$ & 1690 & $1980^{*}$ \\
\hline $\mathrm{t}_{B M O}(\mathrm{Myr})$ & 1590 & 2370 & 1630 & 2420 & 1540 & 2310 & 1530 & 2290 & 2430 & 3350 & 2510 & 3440 & 2330 & 3230 & 2300 & 3190 \\
\hline $\mathrm{T}_{C M B}^{f i n a l}(\mathrm{~K})$ & 3668 & 3684 & 3690 & 3705 & 3638 & 3654 & 3630 & 3645 & 3908 & 3864 & 3943 & 3894 & 3862 & 3823 & 3848 & 3811 \\
\hline \multicolumn{17}{|c|}{ Cooling history model: $\bullet \mathrm{Q}_{B M O}^{\text {present }}=18 \mathrm{TW}, \mathrm{Q}_{r}=8 \mathrm{TW}, \mathrm{Q}_{C M B}^{\text {present }}=10 \mathrm{TW}$} \\
\hline $\mathrm{E}_{\Phi}$ & 284 & 217 & 347 & 263 & 1 & -31 & -7 & -35 & 267 & 183 & 322 & 220 & -43 & -81 & -48 & -84 \\
\hline $\mathrm{t}_{X}(\mathrm{Myr})$ & 1090 & 1460 & 1120 & 1490 & 1770 & 2730 & 1750 & 2700 & 1610 & 1960 & 1670 & 2020 & 2780 & 3920 & 2730 & 3850 \\
\hline $\mathrm{t}_{\Phi}(\mathrm{Myr})$ & 2290 & 2940 & 2470 & 3220 & 1780 & $2360^{*}$ & $1710^{*}$ & $2270^{*}$ & 3050 & 3330 & 3370 & 3640 & $2400^{*}$ & $2740^{*}$ & $2290^{*}$ & $2630^{*}$ \\
\hline $\mathrm{t}_{B M O}(\mathrm{Myr})$ & 2570 & 4490 & 2640 & & 2480 & 4320 & 2450 & 4270 & 4410 & & & & 4170 & & 4100 & \\
\hline $\mathrm{T}_{C M B}^{\text {final }}(\mathrm{K})$ & 4342 & 4399 & 4363 & 4418 & 4312 & 4366 & 4303 & 4357 & 4583 & 4502 & 4613 & 4525 & 4536 & 4471 & 4522 & 4460 \\
\hline \multicolumn{17}{|c|}{ Cooling history model: $\bullet \mathrm{Q}_{B M O}^{\text {present }}=21 \mathrm{TW}, \mathrm{Q}_{r}=8 \mathrm{TW}, \mathrm{Q}_{C M B}^{\text {present }}=13 \mathrm{TW}$} \\
\hline $\mathrm{E}_{\Phi}$ & 488 & 390 & 592 & 464 & 60 & 25 & 45 & 15 & 517 & 398 & 623 & 470 & 30 & -14 & 19 & -22 \\
\hline $\mathrm{t}_{X}(\mathrm{Myr})$ & 740 & 950 & 760 & 970 & 1150 & 1610 & 1140 & 1600 & 1060 & 1240 & 1090 & 1270 & 1680 & 2160 & 1650 & 2140 \\
\hline $\mathrm{t}_{\Phi}(\mathrm{Myr})$ & 1550 & 2100 & 1600 & 2240 & 1310 & 1720 & 1270 & 1670 & 2220 & 2530 & 2390 & 2730 & 1780 & $2090^{*}$ & 1720 & $2030^{*}$ \\
\hline $\mathrm{t}_{B M O}(\mathrm{Myr})$ & 1580 & 2360 & 1620 & 2410 & 1540 & 2300 & 1520 & 2280 & 2420 & 3340 & 2500 & 3430 & 2320 & 3220 & 2290 & 3180 \\
\hline $\mathrm{T}_{C M B}^{\text {final }}(\mathrm{K})$ & 3671 & 3686 & 3693 & 3707 & 3642 & 3657 & 3633 & 3648 & 3911 & 3866 & 3946 & 3896 & 3865 & 3825 & 3851 & 3812 \\
\hline \multicolumn{17}{|c|}{ Cooling history model: $\Delta \mathrm{Q}_{B M O}^{\text {present }}=21 \mathrm{TW}, \mathrm{Q}_{r}=12 \mathrm{TW}, \mathrm{Q}_{C M B}^{\text {present }}=9 \mathrm{TW}$} \\
\hline $\mathrm{E}_{\Phi}$ & 303 & 234 & 365 & 275 & 5 & -28 & -2 & -32 & 280 & 196 & 332 & 230 & -39 & -79 & -45 & -81 \\
\hline $\mathrm{t}_{X}(\mathrm{Myr})$ & 1100 & 1480 & 1130 & 1520 & 1810 & 2820 & 1790 & 2790 & 1650 & 2010 & 1710 & 2070 & 2870 & 4090 & 2820 & 4020 \\
\hline $\mathrm{t}_{\Phi}(\mathrm{Myr})$ & 2340 & 3040 & 2530 & 3310 & 1850 & $2480^{*}$ & $1780^{*}$ & $2400^{*}$ & 3150 & 3470 & 3470 & 3780 & $2510^{*}$ & $2900^{*}$ & $2410^{*}$ & $2800^{*}$ \\
\hline $\mathrm{t}_{B M O}(\mathrm{Myr})$ & 2640 & & 2720 & & 2550 & & 2520 & 4480 & & & & & 4360 & & 4290 & \\
\hline $\mathrm{T}_{C M B}^{\text {final }}(\mathrm{K})$ & 4379 & 4432 & 4401 & 4452 & 4350 & 4404 & 4342 & 4396 & 4617 & 4533 & 4644 & 4556 & 4575 & 4503 & 4561 & 4492 \\
\hline
\end{tabular}

${ }^{*}$ represents the last instance in time when $E_{\Phi}>0$. 
day value of 15, 18, and 21 TW shown as solid curves in Figure 2. Thus, the core heat flow across the CMB, $Q_{c m b}$, at the present day spans values between 9-14 TW, which is the difference between $Q_{B M O}$ and $Q_{r}$ for the cooling histories considered in Table 1. The differences between $Q_{B M O}$ and $Q_{r}$ for the various combinations also imply higher or lower secular cooling rates for the mantle, as the larger value chosen for $Q_{r}$ leaves a smaller amount of the available $12 \mathrm{TW}$ for heating the mantle above the BMO. For example, $Q_{r}$ of $8 \mathrm{TW}$ in the BMO leaves only $4 \mathrm{TW}$ in the solid mantle, leading to faster secular cooling of the mantle which would presumably drive faster secular cooling of the core, and hence this value of $Q_{r}$ is used in combination with larger $Q_{B M O}$ values of $18 \mathrm{TW}$ and $21 \mathrm{TW}$, corresponding to $Q_{c m b}$ values of 10 and $13 \mathrm{TW}$, respectively.

\subsection{Entropy budget}

As mentioned above, the energy budget alone provides enough information to determine the thermal evolution of the BMO layer. However, in order to fully characterize its magnetic evolution and, ultimately, determine the feasibility of dynamo activity, the entropy balance equations are required. Most importantly, the entropy associated with thermal conduction down an adiabat, $E_{\kappa}$, and the Ohmic heating play crucial roles in determining if a dynamo is energetically favorable once all sources of entropy are considered. An equation analogous to 1 can be written for the entropy budget identifying both sources and sinks, and cases for the core have been extensively derived elsewhere (Gubbins et al., 2003, 2004; Nimmo, 2015). Below, we only present their final formulations and describe any changes made in adapting them to 


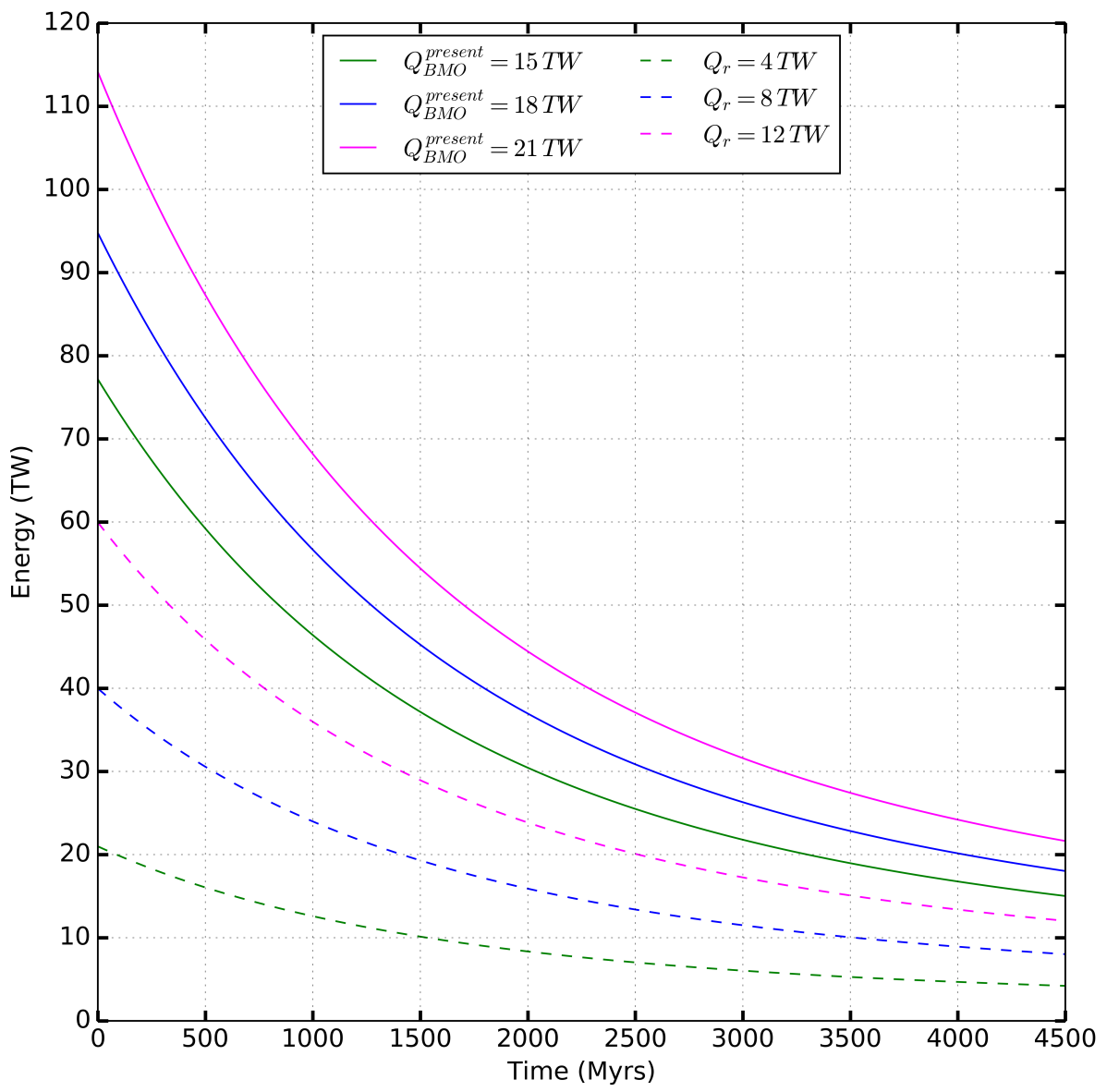

Figure 2: Cooling histories. The three different cooling histories imposed for all model runs (\#1-16 in Table 2) representing 15, 18, and 21 TW present-day, adiabatic heat flux across the top of the BMO layer are shown as solid lines. Dashed lines show the three different radiogenic heating curves 4, 8, and 12 TW resulting from assuming that roughly $30 \%, 70 \%$, and $100 \%$ of the BSE radioactive element budget are initially sequestered within the BMO layer. 
describe our model. The entropy budget is as follows

$$
E_{s}+E_{g}+E_{r}+E_{L}+E_{P}+E_{H}=E_{k}+E_{\Phi}
$$

where $E_{s}, E_{g}, E_{r}, E_{L}, E_{P}$, and $E_{H}$ are the sources and $E_{k}, E_{\Phi}$ are the sinks; $k$ is the thermal conductivity of the layer, and $\Phi$ represents the combined viscous and Ohmic dissipation, though the former is assumed to be negligible (Nimmo, 2015). The small contributions from $E_{P}$ and $E_{H}$ are also ignored in this work. Excluding these terms, the analytical expression for the entropy budget is as the following

$$
\begin{aligned}
& -\int \rho C_{p}\left(\frac{1}{T_{c}}-\frac{1}{T}\right) \frac{d T_{r}^{B M O}}{d t} d V+\frac{Q_{g}}{T}+\int \rho h\left(\frac{1}{T_{c}}-\frac{1}{T}\right) d V \\
& -\frac{4 \pi r_{i}^{2} L_{H}\left(T_{i}-T_{c}\right)}{(d T m / d P-d T / d P) T_{c}^{2} g} \frac{d T_{r}^{B M O}}{d t}=\int k\left(\frac{\nabla T}{T}\right)^{2} d V+\int \frac{\Phi}{T} d V,
\end{aligned}
$$

which is comparable to 8 except for the Carnot efficiency term, $\left(1 / T_{c}-1 / T\right)$.

The criteria of $E_{\Phi}>0$ is commonly used for determining whether the model can generate a dynamo subject to the same assumptions that govern the applicability of this approach to core dynamics (Gubbins et al., 2004; Nimmo, 2015; Buffett et al., 1996; Labrosse, 2003), that the fluid is electrically conductive, rapidly rotating, and undergoing vigorous convection to remain adiabatic and homogenous, all of which are also appropriate for the scenario of a basal magma ocean. This framework is easily adaptable to determining the Ohmic dissipation within a BMO layer. The expression for the first three terms remain the same but the integration bounds must be adapted to encompass the evolving thickness of the BMO layer. However, the term for entropy production due to the release of latent heat at the interface, $E_{L}$, which depends on the cooling rate of the layer and the difference 
between the slopes of the adiabat and the melting curve, is different to the analogous core case.

For the case of the inner core growing outward, the adiabat and the melting curve are anchored at two distinct temperatures; the CMB temperature for the adiabat and the interface (inner-core boundary (ICB)) temperature for the liquidus. However, for the BMO layer crystallizing downward towards the CMB, both the adiabat and melting curve are anchored at the same point. This results in $\left(T_{i}-T_{c}\right)$ in $E_{L}$ to be zero. Another way to think about this is to consider where the latent heat is being generated. In the case of the core, latent heat is released at the ICB which drives convective motions throughout the liquid outer core directly above; but for a BMO layer, the latent heat is generated at the top of the liquid layer, so it does not contribute to convection in the liquid below. Finally, it is clear from these equations that aside from requiring entropy sources to be sufficiently energetic to power the dynamo, $E_{k}$ cannot be too large (i.e. large values of $k$ ) as this would result in most of the entropy being conducted away along the adiabat and not be available to power a dynamo.

\section{Results}

A total of 112 models were simulated, but only 61 models fully crystallized their BMO layer during the age of the Earth. The input parameters for all model runs and their diagnostic outputs are provided (see Table 1 and 2, respectively). The final CMB temperature for all models that fully crystallized during the age of the Earth falls within the plausible range for present-day best-estimate for inferred temperatures at the CMB (Anzellini 
et al., 2013) as shown in Figure 3.

The longevity of the BMO layer varies greatly between models, ranging from short-lived layers crystallizing in 1.5 Gyrs to long-lived layers taking as much as 4.5 Gyrs to fully crystallize. The dominant parameters controlling the thermal evolution of the BMO layer are the imposed depression on the melting curve and the adiabatic gradient; these effects are shown schematically by arrows in Figure 3. For a given cooling history, imposing a larger melting-point depression extends the life of the BMO layer but it has a negligible effect on the final CMB temperature. However, introducing a steeper adiabatic gradient $(d T a / d r=1 \mathrm{~K} / \mathrm{km}$ for filled marker vs $d T a / d r=1.2$ $\mathrm{K} / \mathrm{km}$ for unfilled markers in Figure 3) not only extends the time it takes the BMO layer to fully crystallize, but it also results in a hotter present-day CMB temperature. Changing both parameters simultaneously appears to have an almost linear additive effect.

The general trend observed in Figure 3 is primarily controlled by the total heat budget available to drive the BMO evolution, as defined by the particular cooling history imposed (i.e. combination of $Q_{B M O}^{\text {present }}$ and $Q_{r}$ from Figure 2). Indeed, the fastest cooling models, those on the bottom left, have a bigger heat budget than the slower cooling models on the top right. Moreover, our choice of curves for $Q_{B M O}$ and $Q_{r}$ and their inherent curvatures causes the effects due to melting-point depression and adiabatic gradient to be more pronounced on the slower cooling models, with some of these taking as much as 4.5 Gyrs to fully crystallize. A batch of 32 models with $Q_{B M O}$ $=12 \mathrm{TW}$ and $Q_{r}=4$ and $8 \mathrm{TW}$ (corresponding to $\mathrm{Q}_{C M B}=8$ and $4 \mathrm{TW}$, respectively) were probed and none successfully crystallized the entirety of 
their BMO layer during the 4.5 Gyrs time window. Indeed, 19 other models with the cooling histories reported here were also unsuccessful (see Table 2).

Given the large group of successful models, we focus on two models which best represent the extensive range of evolution scenarios generated by our choices of parameters. Moreover, these reference models resemble the model proposed by (Ziegler and Stegman, 2013). The complete temperature, energy, and entropy evolution for models \#1 and \#15 are respectively shown in the top, middle, and bottom panels of Figure 4.

The evolution of the temperature and the interface radius between the liquid, crystallizing BMO layer and the solid mantle above for each model is shown in Figure 4A and B. Both models share the same imposed cooling history with $\mathrm{Q}_{B M O}=15 \mathrm{TW}$ and $\mathrm{Q}_{r}=4 \mathrm{TW}$, but have two different $d T a / d r$ values. The initial thickness of the BMO (i.e. interface radius) is defined by the intersection of the melting curve (solid black line) with the adiabat (see Figure 1), and its evolution is controlled by the cooling rate of the layer which is directly dominated by the amount of heat being extracted from the layer, as prescribed by the cooling history curve. As the layer cools over time, the adiabat evolves to lower temperatures (shown as colored dashed lines) intersecting the melting curve at greater depths causing the interface radius to decrease and the liquid layer to shrink towards the CMB. Indeed, the retarding effect $d T a / d r$ has on the evolution of the layer (shown in Figure 3 ) is evident here as model \#1 crystallizes about 1 Gyr sooner than model \#15 with the steeper adiabatic gradient.

All the terms in the energy budget outlined in Eq. 8 for both models including an imposed core cooling (yellow curve) term are shown in Figure 


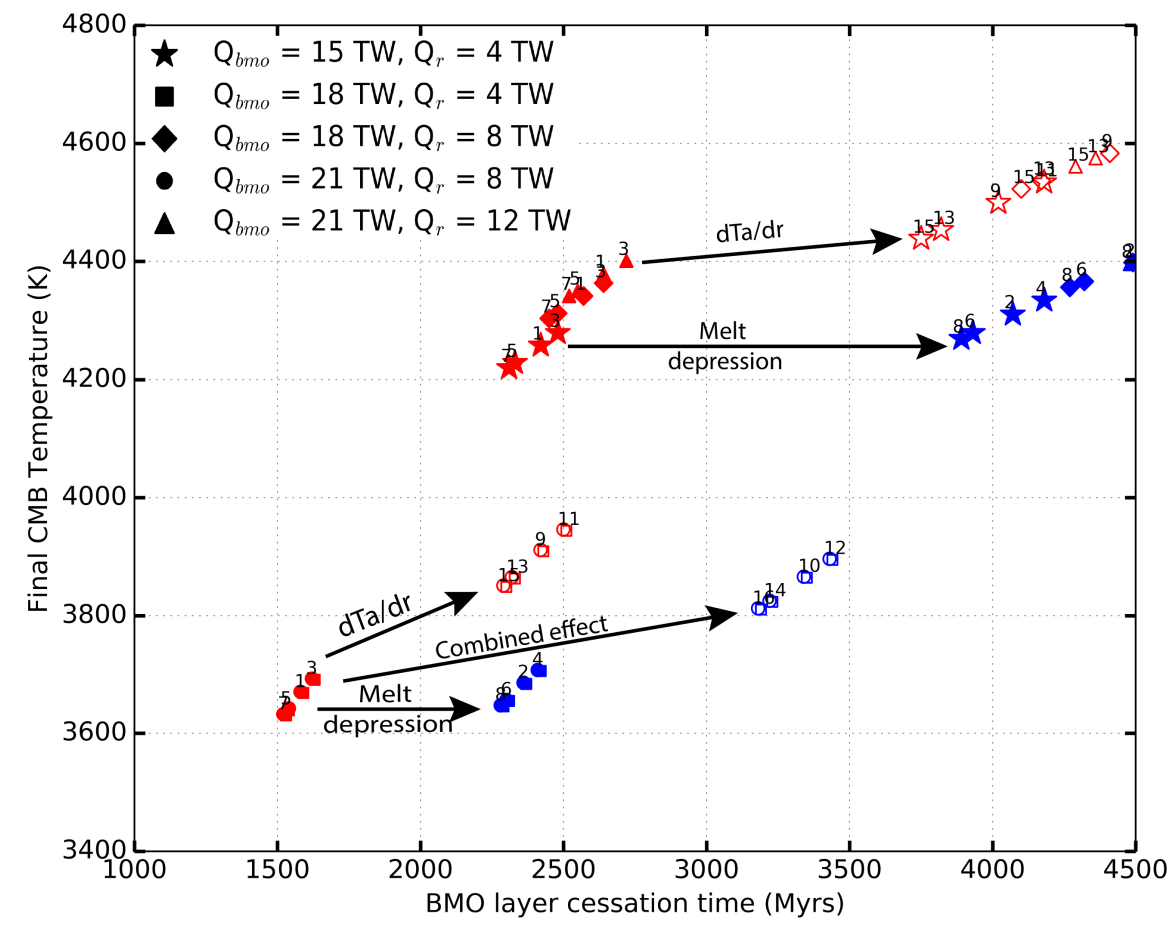

Figure 3: Final CMB temperature. The present-day CMB temperature for all models that successfully crystallized during the age of the Earth are plotted at their corresponding crystallization time. Both adiabatic gradients, $d T a / d r=1$ (filled markers) and $d T a / d r=1.2$ (unfilled markers) and both melting point depressions to the melting curve, $700 \mathrm{~K}$ (red) and $1100 \mathrm{~K}$ (blue) are shown. Schematic arrows show the effect of either parameter in the longevity of the BMO layer for any model run (depicted by the model run numbers) within a given model setup (markers in legend). 
4C and D. The most energetic source during the evolution of the BMO layer is the latent heat released as the layer crystallizes, while gravitational rearrangement and secular cooling terms are small. The amount of latent heat released is the largest during the first billion years of evolution as this is when the crystallization rate is the fastest before being retarded by the imposed melting-point depression on the melting curve. When the layer crystallizes to a thickness of $\approx 200-300 \mathrm{~km}$, the latent heat term becomes comparable to the radiogenic heating and the crystallization rate decreases significantly. Once the layer reaches the $\mathrm{CMB}$, the radiogenic elements are assumed to be trapped in a thin layer atop the $\mathrm{CMB}$, while $Q_{L}$ and $Q_{g}$ terms become zero which results in a corresponding jump in core heat flow. The remaining thermal evolution is primarily accommodated by secular cooling of the core according to the prescribed thermal history model $\left(Q_{B M O}\right)$ and the assumed heat capacity of the core, resulting in the final $T_{C M B}$ values shown in Figure 3. 

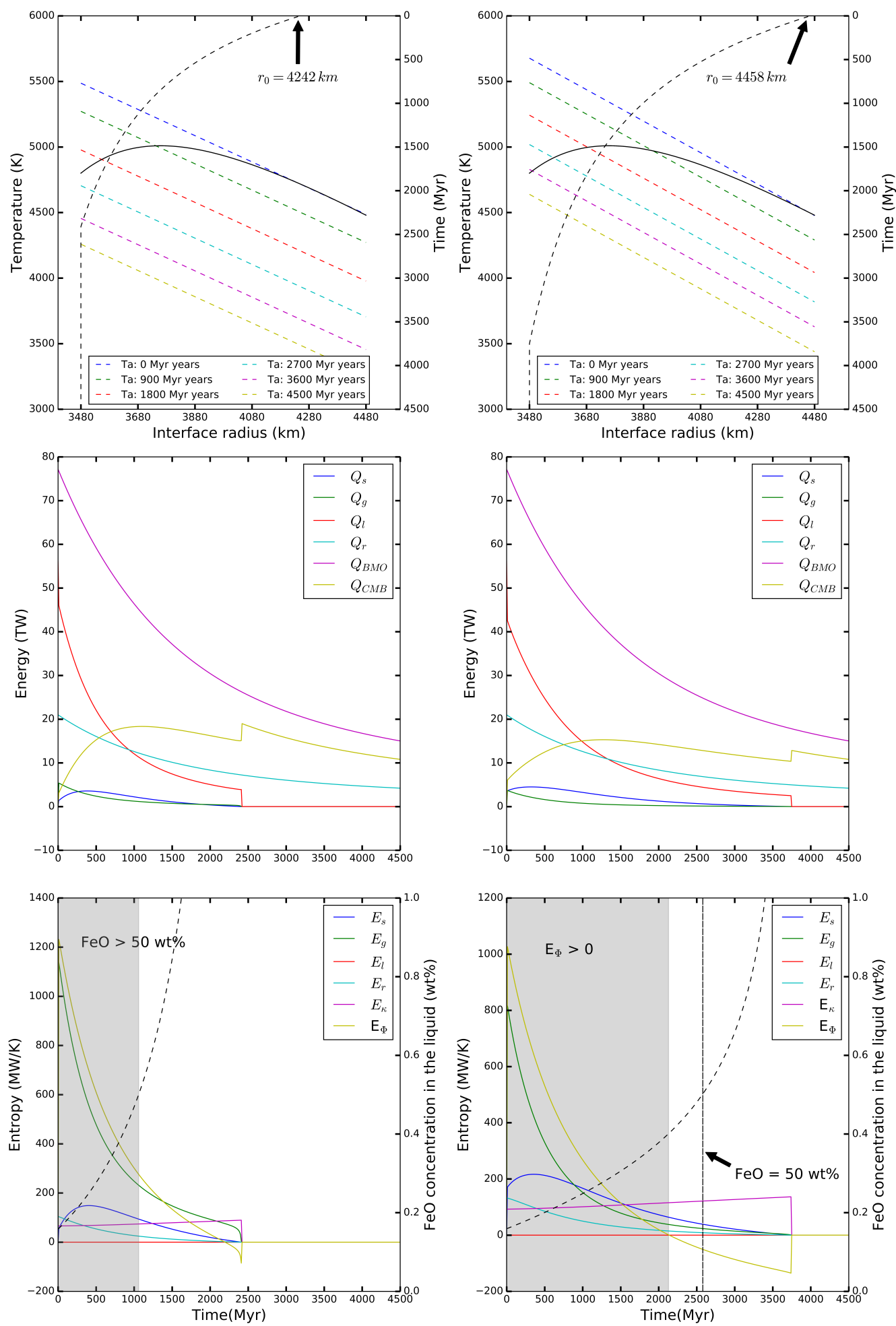
Figure 4: BMO layer evolution. Representative models with $\mathrm{Q}_{B M O}=15 \mathrm{TW}$ and $\mathrm{Q}_{r}=4 \mathrm{TW}$ showing the temperature, energy, and entropy evolution of a 762-km and 978-km thick BMO layer for Models \#1 (left column) and Model \#15 (right columb), respectively. The evolution of the temperature and thickness of the BMO layer over time controlled by the different adiabatic gradients $(d T a / d r=1 \mathrm{~K} / \mathrm{km}$ for Model \#1 and $d T a / d r=1.2 \mathrm{~K} / \mathrm{km}$ for Model \#15) are shown on panel A and B, respectively. Each term in the energy budget outlined in Eq. 1 is plotted in C and D for both models over time. The evolution of the associated entropy terms (solid curves) and evolution of the FeO concentration (dashed curve) over time are shown in $\mathrm{E}$ and $\mathrm{F}$. Shaded regions show the time interval where $E_{\Phi}>0$ while the FeO concentration in the liquid is below $50 \mathrm{wt} \%$ (panel $\mathrm{E}$ ), and last instance when $E_{\Phi}>0$ in panel $\mathrm{F}$ (i.e. when FeO

concentration in run $\# 15$ reaches $\left.50 \%, \mathrm{E}_{\Phi}<0\right)$.

The terms in the entropy budget outlined in Eq. 9 for each model are shown in Figure 4E and F, along with the corresponding evolution of the $\mathrm{FeO}$ concentration in the liquid layer. As described in the Methods section, the latent heat term is zero for the scenario being considered here. While the gravitational term is small in the energy budget, it is the main contributor of entropy to the system (green curve), with the secular (blue curve) and radiogenic (light blue curve) terms contributing marginally. The cumulative total of these entropy sources is balanced against both thermal conduction (magenta curve) and Ohmic dissipation (yellow curve), which are the entropy sinks in the system.

The entropy of thermal conduction is approximately constant and scales with the choice of thermal conductivity, which is $8 \mathrm{~W} / \mathrm{m} / \mathrm{K}$ for these models. Both models sustain $E_{\Phi}>0$ for the first $\approx 2$ billions years, indicating a dynamo would be present in both models over that period of time. However, for BMO dynamos, we consider an additional criteria of whether $D_{\mathrm{FeO}}$ is still consistent with the system once it has become highly enriched in Fe. Consequently, we adopt a value of $c_{F e O}^{l}=50 w t \%$ for this threshold, which 
is shown in the shaded regions. Model \#15 (Figure $4 \mathrm{~F}$ ) falls below $E_{\Phi}=0$ before this threshold is reached, and for such models as this, we report their dynamo cessation time (starting from $\mathrm{t}_{\Phi}=0$ ) as the last instance when $E_{\Phi}>$ 0. In contrast, Model \#1 (Figure $4 \mathrm{E}$ ) reaches the threshold while $E_{\Phi}$ is still positive, and for models that encounter this situation, we report the time they reach this threshold as their dynamo cessation.

We consider this threshold value as a conservative estimate given that $E_{\Phi}$ is well above zero when this point is reached and it would be likely for a dynamo to be operating beyond this time. The partitioning of Fe into the remaining liquid at the solidification front is the mechanism for generating gravitational entropy, which is the dominant term in the entropy budget and controls the magnitude of the Ohmic dissipation. The rate at which the fluid is enriched with Fe, and speed at which the threshold value is reached, is determined by the value of $D_{F e O}$. Model \#1, with $D_{F e O}=0.1$, results in $c_{F e O}^{l}$ increasing more rapidly (dashed line in Figure 4E) than measured increases of $c_{F e O}^{l}$ in Model \#15, with $D_{F e O}=0.5$ (dashed line in Figure 4F). Therefore, the dynamo generated by model \#1 (Figure 4E) is roughly $20 \%$ more energetic than that of model \#15 (Figure 4F).

The amount of entropy available to sustain a dynamo varies among all successful models. Model results for all runs including the available entropy (at $c_{F e O}^{l}=50 w t \%$ ), their corresponding dynamo and BMO cessation times, and their final CMB temperatures are shown in Table 2. Figure 5 shows the amount of Ohmic dissipation at the time the BMO composition encountered the threshold value of $c_{F e O}^{l}=50 w t \%$ for all models that fully crystallized within the age of the Earth. The two clear populations of models shown 
in Figure 5 are primarily controlled by the two, end-member values for the partition coefficient of FeO. Models with $D_{F e O}=0.1$ (Nomura et al., 2011) give rise to long-lived dynamos with lower Ohmic dissipation while those with $D_{F e O}=0.5$ (Andrault et al., 2012) result in dynamos that are shortlived and larger values of $E_{\Phi}$. Both scenarios, however, result in models with sufficiently large $E_{\Phi}$ values for the first 1.5 Gyrs -implying an active dynamo during this time.

Combining our results from Figure 3 for how long it takes the BMO layer to fully crystallize and, in each of those models, how long a dynamo would be active from Figure 5, we can see there exists a wide range of scenarios for a BMO dynamo as shown in Figure 6. These scenarios can be summarized in four distinct populations: Short-lived BMO with (1) short-lived, intense dynamo, and (2) long-lived, weaker dynamo; and long-lived BMO with (3) short-lived, intense dynamo, and (4) long-lived, weaker dynamo.

The intensity of the dynamo is regulated by the value of $D_{\mathrm{FeO}}$ while the time required for the layer to completely solidify is controlled by both melting-point depression and the choice of adiabatic gradient. Those models with the smaller melting-point depression (e.g. $T_{m}^{0}=700 \mathrm{~K}$ ) and a partition coefficient $D_{\mathrm{FeO}}=0.1$ result in short-lived BMO layers with roughly $60 \%$ more entropy available (at $c_{\mathrm{FeO}}^{l}=50 w t \%$ ) to sustain a dynamo compared to the cases with $D_{F e O}=0.5$. A smaller $D_{F e O}$ value results in faster $\mathrm{Fe}$ enrichment of the liquid layer and correspondingly higher values of entropy generated due to the larger density jump at the interface (e.g. larger $E_{g}$ term). Indeed, extending the timescale for solidification of the BMO, by imposing a larger melting-point depression, increases the amount of entropy 


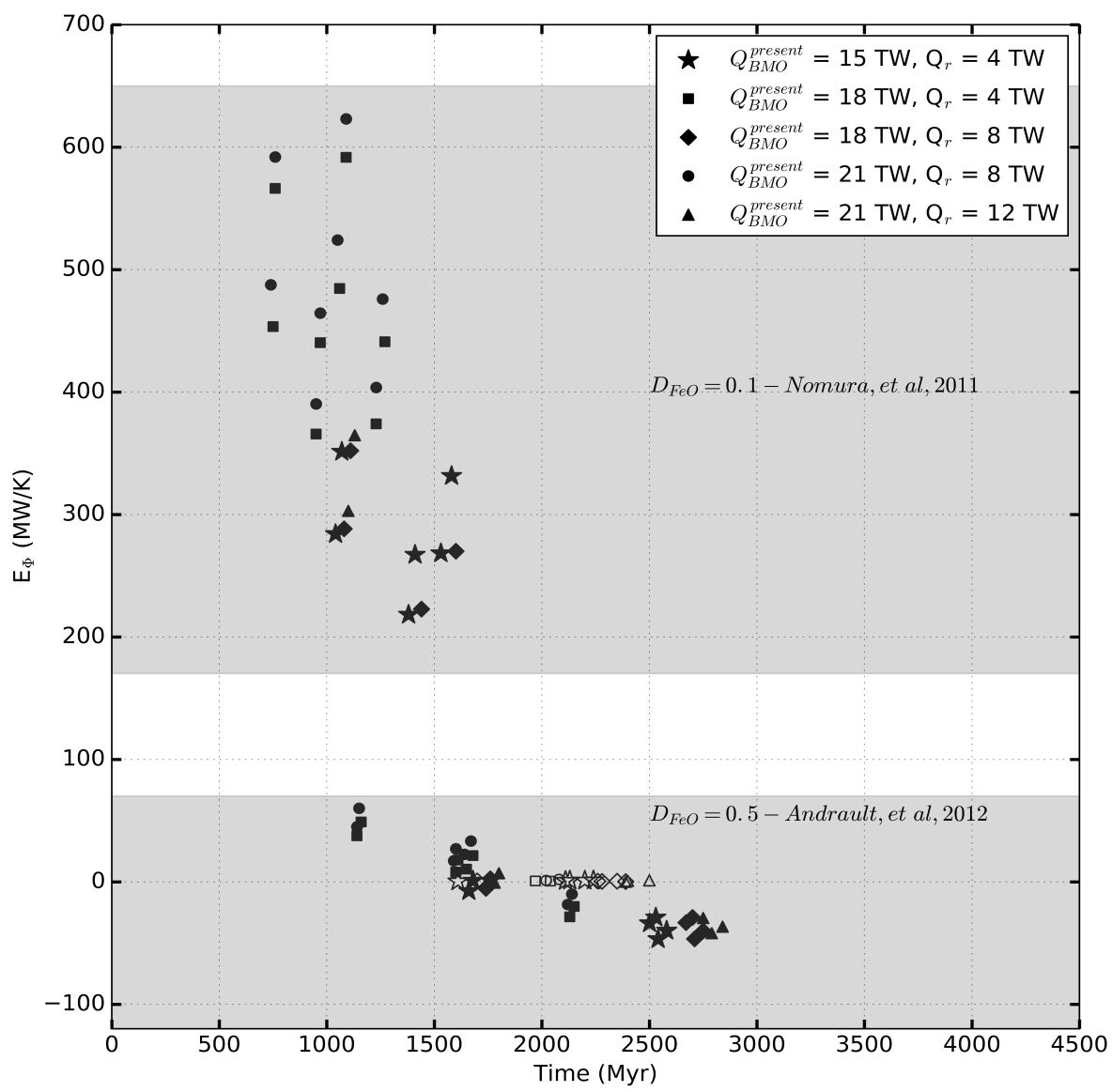

Figure 5: Entropy of Ohmic dissipation over time for all models (at $c_{F e O}^{l}=50 w t \%$ ) whose BMO layer fully crystallized during the age of the Earth plotted in black (filled). For models with $E_{\Phi}<0$ at this cutoff, their entropy value is plotted at the last time when it was positive in black (unfilled). Markers indicate the five difference $\mathrm{Q}_{B M O}-\mathrm{Q}_{r}$ scenarios imposed as described in the text. Two distinct clusters, indicated by the shaded regions, capture the influence of the two different partition coefficient values. 
available to sustain magnetic activity for a longer period of time. It is plausible to have models extending the entire shaded area in Figure 6 through a different combination of allowable model parameters and a less conservative cutoff (i.e. $>c_{F e O}^{l}=50 w t \%$ ).

\section{Discussion}

The present-day CMB temperature for all successful models shown here (Figure 3) falls within a plausible value $(4000 \pm 500 \mathrm{~K})$ for the best estimates up to date (Anzellini et al., 2013). The thermal evolution of a BMO layer atop the liquid core over time is heavily dominated by two parameters: the adiabatic gradient in the liquid, and the melting curve for a lower mantle composition; most importantly how depressed this curve becomes at nearCMB pressures and temperatures.

The entropy budget of a BMO-powered dynamo are heavily dominated by the choice in partition coefficient for $\mathrm{FeO}$ in the liquid, and, in particular, its evolution as the layer becomes heavily-enriched; such behavior is poorly constrained up to date. We anticipate that $D_{F e O}$ will actually start closer to a value of 0.1 (Nomura et al., 2011) and evolve to larger values as the BMO layer decreases in size, thus we believe our end-member choices for $D_{\mathrm{FeO}}$ bracket the expected behavior. Such evolution would in turn result in highly energetic dynamo for the first few hundred million years, similar to Figure $4 \mathrm{E}$, with sustained Ohmic dissipation values beyond 2 Gyrs, resembling the scenario shown in Figure $4 \mathrm{~F}$ with the higher $D_{F e O}$ value.

Our models successfully show that a crystallizing BMO can be an effective mechanism for generating and sustaining a dynamo throughout early 


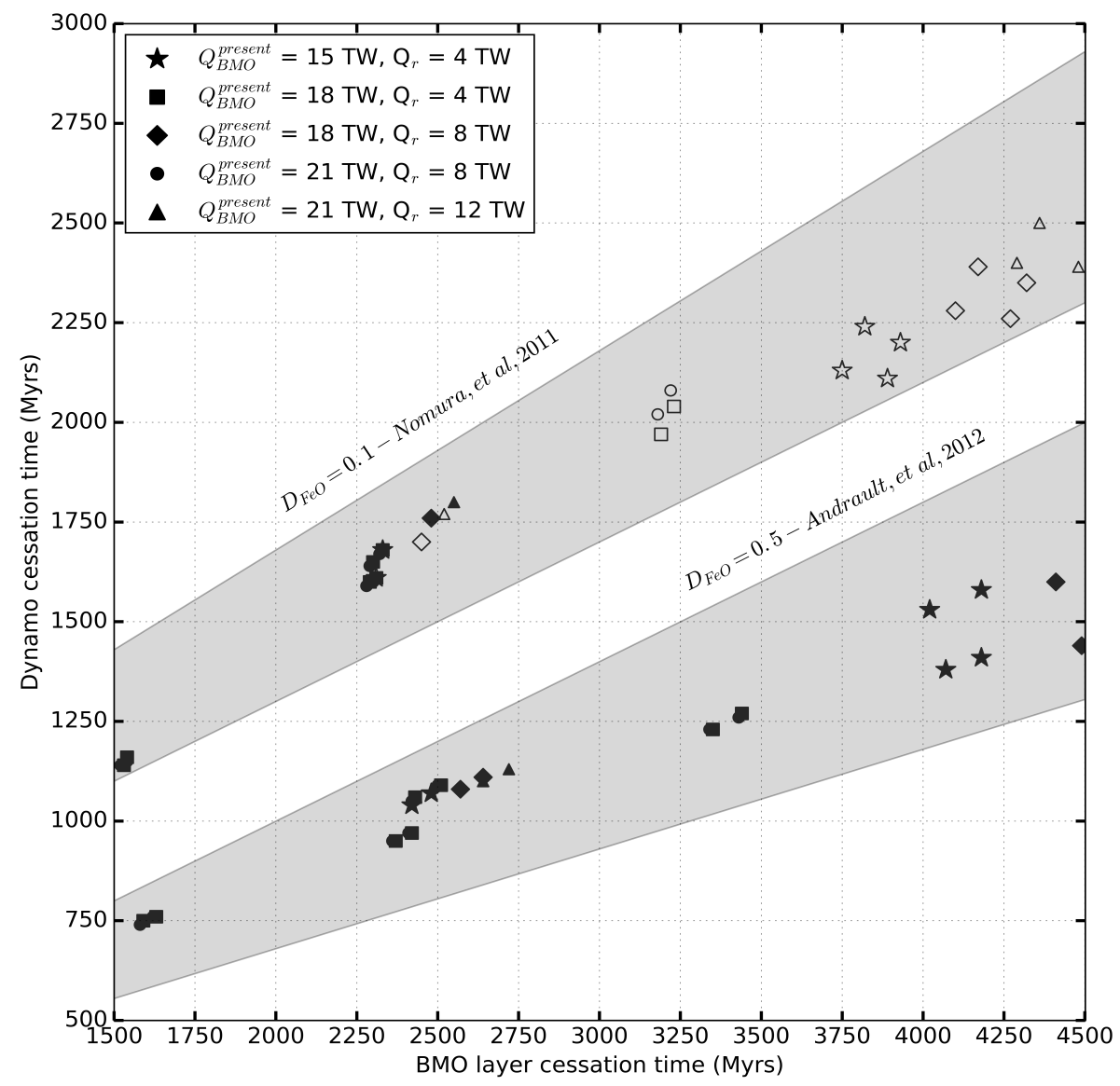

Figure 6: Duration of dynamo activity versus BMO layer crystallization time for all models that fully crystallized. Models with $E_{\Phi}>0$ at $c_{F e O}^{l}=50 w t \%$ are plotted in black (filled), while those whose $E_{\Phi}$ value was negative at $c_{F e O}^{l}=50 w t \%$ are plotted in black (unfilled) at the last time $E_{\Phi}$ was above zero. Shaded regions as per Figure 5. 
Earth. Most importantly, all models whose BMO layer successfully crystallized during the age of the Earth have sufficient entropy to generate magnetic activity for at least the first 1.5 Gyrs (Figure 5), with some lasting well passed 2 Gyrs. A vast range of parameter combinations other than the choices we made would also lead to scenarios with enough entropy to generate a dynamo for at least 1.5 Gyrs, conservatively. This makes a BMO-driven dynamo a plausible mechanism for explaining the existence of a magnetic field early on in Earth's history as required by paleomagnetic observations; thus relaxing the need for a global magnetic field to be entirely powered by thermal cooling of the core. Additionally, since exsolution-based mechanisms are also contingent upon the presence of a BMO of some depth, the BMO-powered dynamo is not mutually exclusive with them and the possibility exists for both to operate, either contemporaneously or sequentially.

The time required to fully crystallize a BMO layer as well as the duration of a dynamo that such evolution would produce varies greatly among models (Figure 6). However, it is possible to have some scenarios where a dynamo can be sustained (e.g. $E_{\Phi}>0$ ) for a longer time window given the conservative cutoff employed here, all while also constraining its BMO layer to be fully crystallized at present day and a CMB temperature in agreement with current best estimates. Previous work emphasized that "thermal catastrophe" outcomes were constraints for model evolutions and used this as a basis for determining which combinations of parameters were deemed successful (Korenaga, 2013, 2008; Driscoll and Bercovici, 2014), however, by these standards all of our models would be unsuccessful which demonstrates this logic is not sound. Instead, we propose the only constraint that must 
be satisfied is that the mantle with an initial BMO must completely solidify within the age of the Earth, and if not, this is what we would refer to as unsuccessful model. There is nothing catastrophic or implausible about thermal evolutions that exceed the solidus temperatures for part of their evolution.

\section{Conclusion}

The evolution of a crystallizing basal magma ocean overlying the liquid core can explain the magnetic evolution of early Earth as most models tested here are energetic enough to sustain a dynamo during this time. Indeed, some of the models sustain dynamos well into the Archaen, though marginally.

The evolution of a basal magma ocean, as modeled here, depends heavily on the material properties of silicates in general and silicate melts in particular. Parameters such as the partitioning coefficient for a molten silicate layer and its evolution as the layer becomes highly enriched in $\mathrm{FeO}$ (i.e. $\left.c_{\mathrm{FeO}}^{l}>50 \mathrm{wt} \%\right)$, as well as the associated melting-point depression of such a composition at near-CMB pressure and temperature conditions are crucial parameters yet they are poorly constrained to date. The work presented here, while it provides a novel mechanism to generate a dynamo and understand Earth's complex magnetic history, should serve as motivation to better constrain these parameters experimentally.

Moreover, the versatility of this model does not hinge on a specific condition of core cooling; in fact, it can be adapted to complement thermal evolution models involving any thermal conductivity values for the core. More importantly, this model complements previous dynamo mechanism proposed by being able to generate enough power to induce and sustain an early dy- 
namo whose lifespan can be extended by different mechanism (e.g. the $\mathrm{Mg}$ exsolution of (O'Rourke et al., 2017)). A similar computational formulation can be relevant to simulate thermal history schemes in "super-Earth" exoplanets.

\section{Acknowledgements}

We acknowledge C. Davies for providing the idea of calculating the entropy budget for the BMO and for providing the code used for (Davies, 2015) for us to adapt. We thank J. Badro, C. Boukare, L. Stixrude, S. Stewart for helpful discussions. We acknowledge support from NSF.

\section{References}

Andrault, D., Petitgirard, S., Nigro, G. L., Devidal, J.-L., Veronesi, G., Garbarino, G., Mezouar, M., 2012. Solid-liquid iron partitioning in Earths deep mantle. Nature 487 (7407), 354.

Anzellini, S., Dewaele, A., Mezouar, M., Loubeyre, P., Morard, G., 2013. Melting of iron at Earths inner core boundary based on fast X-ray diffraction. Science 340 (6131), 464-466.

Badro, J., Aubert, J., Hirose, K., Nomura, R., Blanchard, I., Borensztajn, S., Siebert, J., 2018. Magnesium Partitioning Between Earth's Mantle and Core and its Potential to Drive an Early Exsolution Geodynamo. Geophysical Research Letters 45 (24), 13-240.

Badro, J., Siebert, J., Nimmo, F., 2016. An early geodynamo driven by 
exsolution of mantle components from Earths core. Nature 536 (7616), 326.

Boukaré, C.-E., Ricard, Y., 2017. Modeling phase separation and phase change for magma ocean solidification dynamics. Geochemistry, Geophysics, Geosystems 18 (9), 3385-3404.

Boukaré, C.-E., Ricard, Y., Fiquet, G., 2015. Thermodynamics of the MgOFeO-SiO2 system up to 140 GPa: Application to the crystallization of Earth's magma ocean. Journal of Geophysical Research: Solid Earth $120(9), 6085-6101$.

Buffett, B. A., Huppert, H. E., Lister, J. R., Woods, A. W., 1996. On the thermal evolution of the Earth's core. Journal of Geophysical Research: Solid Earth 101 (B4), 7989-8006.

Canup, R. M., Asphaug, E., 2001. Origin of the Moon in a giant impact near the end of the Earth's formation. Nature 412 (6848), 708.

Caracas, R., Hirose, K., Nomura, R., Ballmer, M. D., 2019. Melt-crystal density crossover in a deep magma ocean. Earth and Planetary Science Letters 516, 202-211.

Ćuk, M., Stewart, S. T., 2012. Making the Moon from a fast-spinning Earth: a giant impact followed by resonant despinning. Science 338 (6110), $1047-$ 1052 .

Davies, C. J., 2015. Cooling history of Earths core with high thermal conductivity. Physics of the Earth and Planetary Interiors 247, 65-79. 
de Koker, N., Steinle-Neumann, G., Vlček, V., 2012. Electrical resistivity and thermal conductivity of liquid Fe alloys at high $\mathrm{P}$ and $\mathrm{T}$, and heat flux in Earths core. Proceedings of the National Academy of Sciences 109 (11), 4070-4073.

De Koker, N., Stixrude, L., 2009. Self-consistent thermodynamic description of silicate liquids, with application to shock melting of $\mathrm{MgO}$ periclase and MgSiO3 perovskite. Geophysical Journal International 178 (1), 162-179.

Driscoll, P., Bercovici, D., 2014. On the thermal and magnetic histories of Earth and Venus: Influences of melting, radioactivity, and conductivity. Physics of the Earth and Planetary Interiors 236, 36-51.

Du, Z., Boujibar, A., Driscoll, P., Fei, Y., 2019. Experimental constrains on an $\mathrm{MgO}$ exsolution-driven geodynamo. Geophysical Research Letters.

Du, Z., Jackson, C., Bennett, N., Driscoll, P., Deng, J., Lee, K. K., Greenberg, E., Prakapenka, V. B., Fei, Y., 2017. Insufficient energy from $\mathrm{MgO}$ exsolution to power early geodynamo. Geophysical Research Letters $44(22), 11-376$.

Dziewonski, A. M., Anderson, D. L., 1981. Preliminary reference Earth model. Physics of the earth and planetary interiors 25 (4), 297-356.

Fiquet, G., Auzende, A., Siebert, J., Corgne, A., Bureau, H., Ozawa, H., Garbarino, G., 2010. Melting of peridotite to 140 gigapascals. Science 329 (5998), 1516-1518.

Gomi, H., Ohta, K., Hirose, K., Labrosse, S., Caracas, R., Verstraete, M. J., Hernlund, J. W., 2013. The high conductivity of iron and thermal evolution 
of the Earths core. Physics of the Earth and Planetary Interiors 224, 88103.

Gubbins, D., Alfe, D., Masters, G., Price, G. D., Gillan, M., 2003. Can the Earths dynamo run on heat alone? Geophysical Journal International $155(2), 609-622$.

Gubbins, D., Alfe, D., Masters, G., Price, G. D., Gillan, M., 2004. Gross thermodynamics of two-component core convection. Geophysical Journal International 157 (3), 1407-1414.

Hirose, K., Morard, G., Sinmyo, R., Umemoto, K., Hernlund, J., Helffrich, G., Labrosse, S., 2017. Crystallization of silicon dioxide and compositional evolution of the Earths core. Nature 543 (7643), 99.

Holmström, E., Stixrude, L., Scipioni, R., Foster, A., 2018. Electronic conductivity of solid and liquid ( $\mathrm{Mg}, \mathrm{Fe}$ ) $\mathrm{O}$ computed from first principles. Earth and Planetary Science Letters 490, 11-19.

Korenaga, J., 2008. Urey ratio and the structure and evolution of Earth's mantle. Reviews of Geophysics 46 (2).

Korenaga, J., 2013. Initiation and evolution of plate tectonics on Earth: theories and observations. Annual review of earth and planetary sciences 41, 117-151.

Labrosse, S., 2003. Thermal and magnetic evolution of the Earths core. Physics of the Earth and Planetary Interiors 140 (1-3), 127-143. 
Labrosse, S., 2015. Thermal evolution of the core with a high thermal conductivity. Physics of the Earth and Planetary Interiors 247, 36-55.

Labrosse, S., Hernlund, J., Coltice, N., 2007. A crystallizing dense magma ocean at the base of the Earths mantle. Nature 450 (7171), 866.

Laneuville, M., Hernlund, J., Labrosse, S., Guttenberg, N., 2018. Crystallization of a compositionally stratified basal magma ocean. Physics of the Earth and Planetary Interiors 276, 86-92.

Lock, S. J., Stewart, S. T., Petaev, M. I., Leinhardt, Z., Mace, M. T., Jacobsen, S. B., Cuk, M., 2018. The origin of the Moon within a terrestrial synestia. Journal of Geophysical Research: Planets 123 (4), 910-951.

McDonough, W. F., Sun, S.-S., 1995. The composition of the Earth. Chemical Geology 120 (3-4), 223-253.

McWilliams, R. S., Spaulding, D. K., Eggert, J. H., Celliers, P. M., Hicks, D. G., Smith, R. F., Collins, G. W., Jeanloz, R., 2012. Phase transformations and metallization of magnesium oxide at high pressure and temperature. Science 338 (6112), 1330-1333.

Nimmo, F., 2015. Energetics of the core: Ed. G. Schubert. Treatise on geophysics (second edition). Elsevier, Oxford 8, 27-55.

Nomura, R., Ozawa, H., Tateno, S., Hirose, K., Hernlund, J., Muto, S., Ishii, H., Hiraoka, N., 2011. Spin crossover and iron-rich silicate melt in the Earths deep mantle. Nature 473 (7346), 199.

Olson, P., 2013. The new core paradox. Science 342 (6157), 431-432. 
O’Rourke, J. G., Korenaga, J., Stevenson, D. J., 2017. Thermal evolution of Earth with magnesium precipitation in the core. Earth and Planetary Science Letters 458, 263-272.

ORourke, J. G., Stevenson, D. J., 2016. Powering Earths dynamo with magnesium precipitation from the core. Nature 529 (7586), 387.

Pozzo, M., Davies, C., Gubbins, D., Alfe, D., 2012. Thermal and electrical conductivity of iron at Earths core conditions. Nature 485 (7398), 355.

Scipioni, R., Stixrude, L., Desjarlais, M. P., 2017. Electrical conductivity of $\mathrm{SiO} 2$ at extreme conditions and planetary dynamos. Proceedings of the National Academy of Sciences 114 (34), 9009-9013.

Soubiran, F., Militzer, B., 2018. Electrical conductivity and magnetic dynamos in magma oceans of Super-Earths. Nature communications 9 (1), 3883.

Spaulding, D., McWilliams, R., Jeanloz, R., Eggert, J., Celliers, P., Hicks, D., Collins, G., Smith, R., 2012. Evidence for a phase transition in silicate melt at extreme pressure and temperature conditions. Physical Review Letters 108 (6), 065701.

Stackhouse, S., Stixrude, L., Karki, B. B., 2010. Thermal conductivity of periclase (MgO) from first principles. Physical review letters 104 (20), 208501.

Stevenson, D. J., 2003. Planetary magnetic fields. Earth and planetary science letters 208 (1-2), 1-11. 
Stixrude, L., 2014. Melting in super-earths. Philosophical Transactions of the Royal Society A: Mathematical, Physical and Engineering Sciences 372 (2014), 20130076.

Stixrude, L., de Koker, N., Sun, N., Mookherjee, M., Karki, B. B., 2009. Thermodynamics of silicate liquids in the deep Earth. Earth and Planetary Science Letters 278 (3-4), 226-232.

Tarduno, J. A., Cottrell, R. D., Davis, W. J., Nimmo, F., Bono, R. K., 2015. A Hadean to Paleoarchean geodynamo recorded by single zircon crystals. Science 349 (6247), 521-524.

Tarduno, J. A., Cottrell, R. D., Watkeys, M. K., Hofmann, A., Doubrovine, P. V., Mamajek, E. E., Liu, D., Sibeck, D. G., Neukirch, L. P., Usui, Y., 2010. Geodynamo, solar wind, and magnetopause 3.4 to 3.45 billion years ago. Science 327 (5970), 1238-1240.

Wolf, A. S., Bower, D. J., 2018. An equation of state for high pressuretemperature liquids (RTpress) with application to MgSiO3 melt. Physics of the Earth and Planetary Interiors 278, 59-74.

Ziegler, L., Stegman, D., 2013. Implications of a long-lived basal magma ocean in generating Earth's ancient magnetic field. Geochemistry, Geophysics, Geosystems 14 (11), 4735-4742. 